\title{
EMH Jahresbericht 2000
}

Ende 1999 wurde an einer Klausurtagung das bestehende Konzept des Schweizerischen Ärzteverlages EMH analysiert und überarbeitet. Die daraus resultierenden Erkenntnisse wurden in der "Vision 2001" festgehalten. Diese wurde im Februar 2000 dem Verwaltungsrat EMH vorgelegt und von diesem einstimmig genehmigt. Anschliessend wurde dieses Papier auch vom Zentralvorstand der FMH zur Kenntnis genommen und gutgeheissen. Ein wichtiger Punkt für die Umsetzung dieses Papiers war das enorme Engagement der früheren und der neuen Zeitschriftenredaktionen bei der Umgestaltung unserer Zeitschriften. Diese Redaktorinnen und Redaktoren haben die Schaffung von optimalen Printprodukten zur standespolitischen Information, medizinischen Fortbildung und wissenschaftlichen Publizistik erst möglich gemacht. Ihnen gebührt unser ganz grosser Dank.

Wesentliche Punkte der "Vision 2001" wurden schon zu früheren Zeitpunkten publiziert, seien hier der Vollständigkeit halber nochmals festgehalten:

- Die Schweizerische Ärztezeitung verzichtet weitgehend auf die Publikation medizinischer Fortbildung und baut ihre Kernthemen wie Standespolitik, Gesundheitsökonomie, Ethik usw. aus.

- Die Schweizerische Medizinische Wochenschrift publiziert ausschliesslich in englischer Sprache und erscheint vierzehntäglich. Ziel ist, bei der SMW die internationale Beachtung und den Impact factor mittelfristig erheblich zu steigern und den wissenschaftlich tätigen Medizinern ein attraktives kontinentaleuropäisches Publikationsorgan anzubieten.

- Seit Januar 2001 erscheint eine neue Fortbildungszeitschrift mit dem Namen "Swiss Medical Forum». Das SMF wird in seinen Hauptteilen in die jeweils andere Landessprache übersetzt und grösstenteils als selbständige deutsch- und französischsprachige Zeitschrift publiziert.

- Für das SMF wird ein Dreijahres-Fortbildungsplan entwickelt, der neben dem Printprodukt in einer zweiten Phase durch interaktives Selfassessment im Internet ergänzt wird.

- Das Schweizerische Medizinische Jahrbuch erscheint auch als elektronisches Nachschlagewerk im Internet.

- Der Zentralvorstand der FMH anerkennt, dass durch dieses neue Konzept von EMH ein wesentlich grösserer Nutzen für ihre Mitglieder entsteht. Die FMH verzichtet im Jahr 2001 auf einen Teil ihrer Lizenzabgabe aus der Schweizerischen Ärztezeitung respektive erhebt bei ihren Mitgliedern einen Sonderbeitrag zur Finanzierung eines Teils der dafür entstehenden Kosten.

- Im elektronischen Bereich wird der Aufbau eines auf die Schweizerische Ärzteschaft zugeschnittenen Internetportals in Angriff genommen.

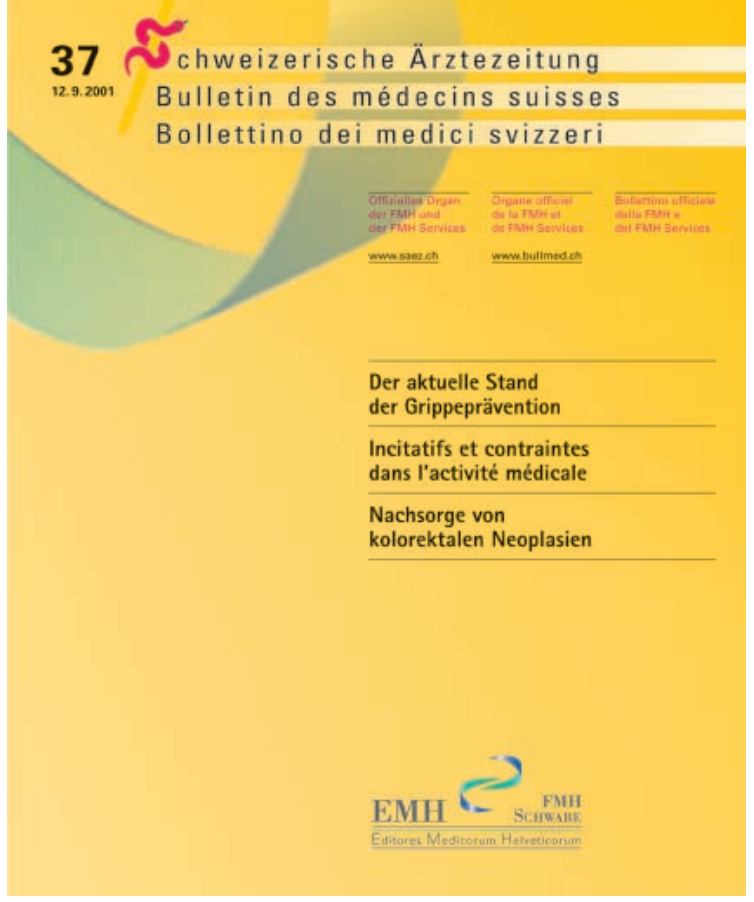

\section{Schweizerische Ärztezeitung}

Die SÄZ kommt nicht zur Ruh! Seit ihrem Erscheinen im Schweizerischen Ärzteverlag muss das Zeitschriftenkonzept laufend überprüft und neuen Anforderungen angepasst werden. Die Berichtsperiode ist gekennzeichnet durch das zunehmende und überaus berechtigte Bedürfnis der Leserinnen und Leser an aktuellen und zuverlässigen Informationen zur manchmal etwas verwirrenden Berufspolitik. Bewährt hat sich das dezentral arbeitende Redaktionsteam. Chefredaktor Dr. Markus Trutmann stehen Dr. Claude Aubert, Dr. Ludwig T. Heuss, Reto Steiner und Dr. Erhard Taverna als nebenamtliche externe Redaktoren zur Seite. Im Verlag sind Thomas Heuer als koordinierender Redaktor und Margrit Neff im Sekretariat die ordnenden Instanzen dieser lebhaften Zeitschrift. Bewährt hat sich insbesondere auch die redaktionelle Unabhängigkeit der Ärztezeitung gegenüber der Herausgeberin und dem Verlag: Die SÄZ praktiziert eine liberale Redaktionspolitik, welche eine ausgewogene Darstellung sehr verschiedener Meinungen gestattet. Nebst der offiziellen Stimme ist die Meinungsvielfalt ein wesentlicher Bestandteil der FMH, die in der SÄZ ihren Ausdruck finden kann.

Der Verzicht auf die medizinischen Fortbildungsbeiträge ist gleichzeitig eine Chance, die Berufspolitik und ihre "Hilfswissenschaften» wie Recht, Ökonomie, Ethik, Medizingeschichte publizistisch aufzuwerten. An der konkreten Umsetzung, welche für Ende 2001 geplant ist, wird intensiv gearbeitet. 
Seit dem 21. Juni 2000 ist die SÄZ online: sämtliche Artikel erscheinen synchron zur gedruckten Ausgabe im Volltext und sind kostenlos verfügbar. Dankbar sind all jene, die schnell auf ein vollständiges Archiv zurückgreifen, aber zu Hause keine spezielle Ablage dafür reservieren wollen ... Die Möglichkeiten der Interaktionen im elektronischen Forum werden trotz stetig steigender Zugriffszahlen auf der Website noch recht zaghaft genutzt. Trotzdem oder gerade deshalb werden die Editores die Angebote im Bereich Electronic Publishing mit ungebremstem Impetus weiter ausbauen, damit jedes einzelne FMHMitglied das finden wird, was es braucht.

Die SÄZ ist auch wirtschaftlich erfolgreich. Dazu muss konstatiert werden, dass ein substantieller Teil ihrer erwirtschafteten Mittel an die FMH abgegeben wird und dass die Redaktionsmitglieder in zahlreichen anderen Projekten engagiert sind. Die personellen und materiellen Mittel der Redaktion auszubauen, ist eine der Aufgaben der nächsten Jahre.

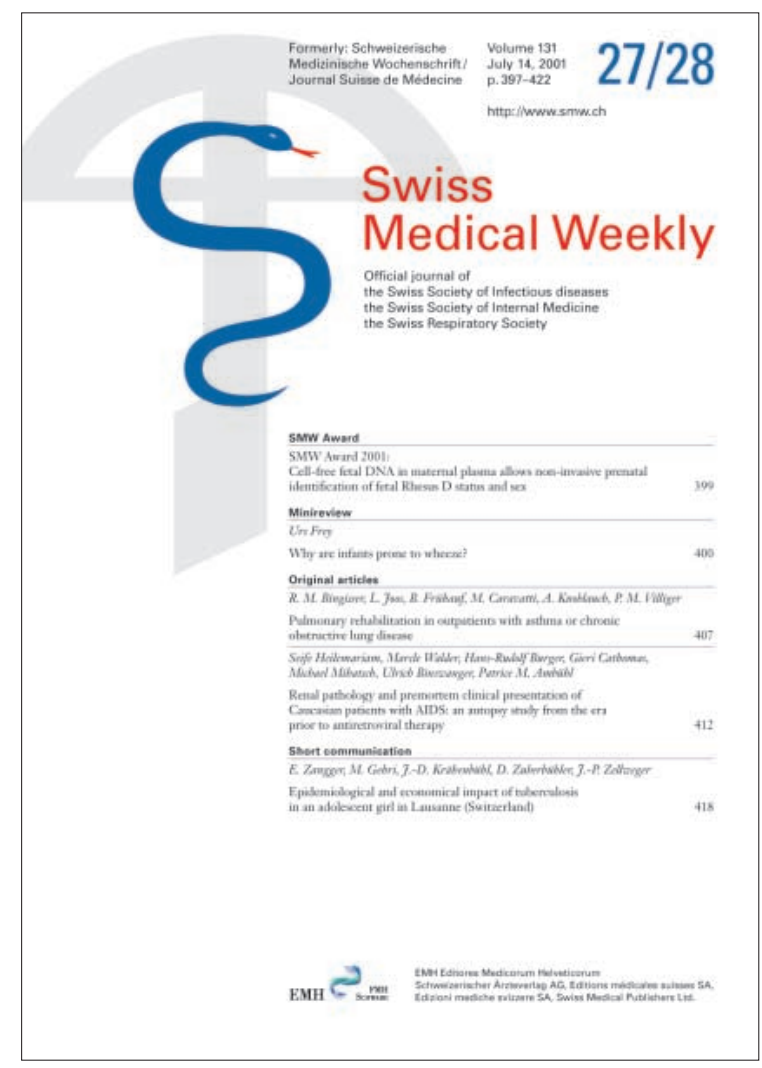

\section{Swiss Medical Weekly}

Der seit vielen Jahren spürbar immer notwendigere Wechsel von einer dreisprachigen Zeitschrift mit einer Mischung von Originalarbeiten und Fortbildung zu einem internationalen wissenschaftlichen Journal in englischer Sprache wurde während des vergangenen Jahres vorbereitet und zum Jahreswechsel vollzogen. Die Redaktion mit Chefredaktor Prof. Andreas Schaffner, Zürich, Prof. Peter Gehr, Bern, Prof. André P. Perruchoud, Basel, Prof. Werner Straub, Bern, Prof.
Peter Suter, Genève, und Prof. Ludwig von Segesser, Lausanne, leistete eine immense Arbeit. Sie wird unterstützt durch einen Statistiker sowie Mediziner und Übersetzer englischer Muttersprache als Lektoren. Im EMH wird die SMW von Dr. Natalie Marty als koordinierender Redaktorin geleitet. Gisela Wagner managt im Sekretariat ein effizientes Peer-reviewSystem.

Jeder Beitrag wird mindestens zwei Reviewern vorgelegt, davon mindestens einem im Ausland, und vom Statistiker durchgesehen. Die Redaktion ist dank elektronischem Datenverkehr laufend in engem Kontakt untereinander.

Der SMW Award wird in diesem Jahr erstmals auch europaweit ausgeschrieben. Die SMW ist bereits seit 1996 online verfügbar.

Auch die neue SMW wird natürlich in der Medline und den anderen wichtigen medizinischen Datenbanken zitiert, neu unter dem Kürzel Swiss Med Wkly.

Die SMW wird aus ökonomischer Sicht klar an Wert verlieren, als wissenschaftliches Publikationsorgan aber eine grosse Wertsteigerung erzielen.

\section{Swiss Medical Forum}

Es brauchte einigen Mut, um im übersättigten Markt der Medizinischen Zeitschriften eine neue, zweisprachige Fortbildungszeitschrift zu lancieren. Den Ausschlag gab das überzeugende Konzept, das eine enge Zusammenarbeit zwischen Praktikern und Spitalärzten vorsah. Dieser Umstand hat zu sehr interes-

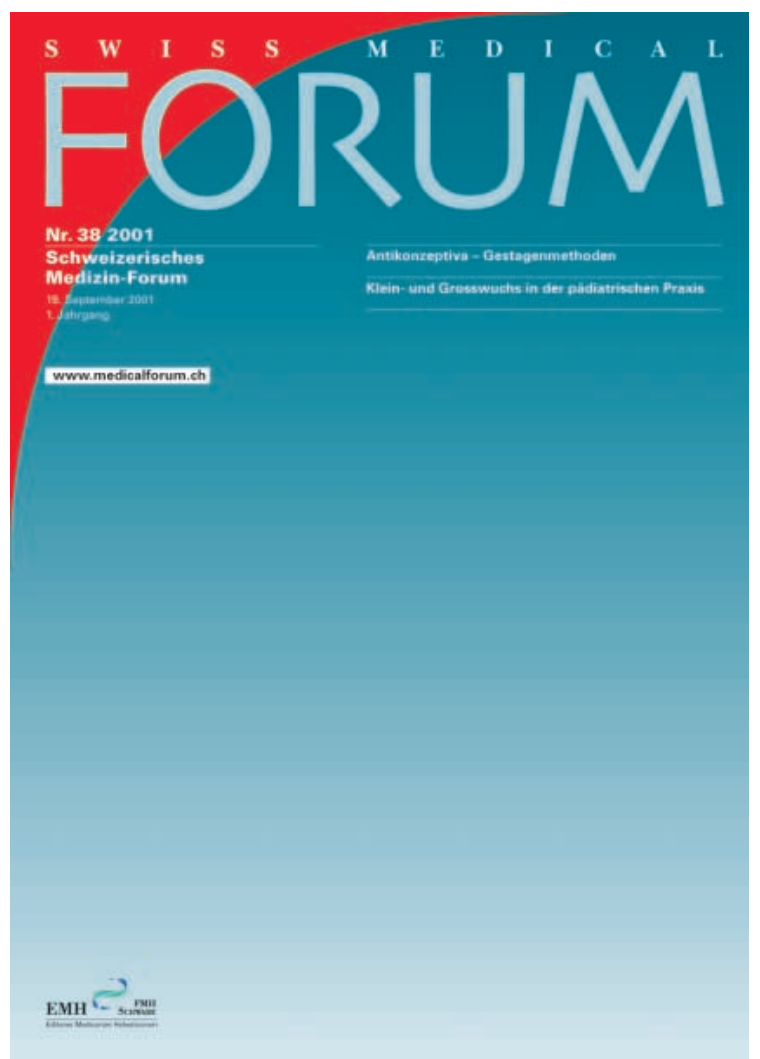


santen Diskussionen und erfreulicherweise zu einer sehr guten und fruchtbaren Zusammenarbeit geführt. Erst die Gründung des Swiss Medical Forum hat es aus ökonomischer Sicht erlaubt, die Veränderungen bei der SMW an die Hand zu nehmen. Aus diesen Gründen liegen die Verlagsrechte dieser Zeitschrift beim Verlag Schwabe, der EMH die Lizenz zur Verfügung stellt wie bei der SMW.

Das Redaktionsteam mit Chefredaktor Prof. Reto Krapf, Dr. Ludwig T. Heuss, Dr. Pierre Périat, Prof. Ruedi Ritz, Prof. Rolf A. Streuli, Prof. Antoine de Torrenté und Prof. Bruno Truniger hat im vergangenen Jahr ein griffiges, spannendes Konzept ausgearbeitet und Kontakte zu Hunderten von Autorinnen und Autoren geknüpft. Das SMF wurde in der Aufbauphase von Dr. Markus Trutmann und Ruth Schindler betreut, später zugestossen ist Dr. Romaine Viollier, welche nun als Managing Editor das Team ergänzt und das tägliche Geschäft im Verlag führt.

Als sehr aufwendig und arbeitsintensiv erwies sich die Publikation des Hauptteils der Zeitschrift in zwei komplett eigenständigen Ausgaben für die deutsch- und die französischsprachige Schweiz. Dr. Markus Trutmann ist es gelungen, ein Übersetzerteam aufzubauen, das in der Lage ist, die komplexen medizinischen Texte zuverlässig in die zweite Landessprache zu übertragen. Die Akzeptanz der neuen Zeitschrift bei den Leserinnen und Lesern ist sehr gut. Die Bereitschaft zur Mitarbeit ist bei allen angefragten Autoren gross. Der nächste Schritt ist die Selbstevaluation via Internet. Die Vorarbeiten dazu laufen auf Hochtouren. Dieses Projekt wird von Dr. Markus Trutmann betreut.

\section{PrimaryCare}

Der Ursprung der Zeitschrift PrimaryCare lag im Wunsch der Schweizerischen Gesellschaft für Allgemeinmedizin (SGAM) nach einer eigenen Zeitschrift, auf die sie Einfluss nehmen kann und in der die Anliegen der Grundversorger gespiegelt werden. Das von EMH daraufhin entworfene Konzept fand schnell die Zustimmung der SGAM. In den weiteren Verhandlungen wirkte sich der gute Kontakt von SGAM und dem Kollegium für Hausarztmedizin (KHM) aus, und so entstand die Grundversorgerzeitschrift PrimaryCare. PrimaryCare ist das offizielle Organ von SGAM und KHM.

Getragen wird die Zeitschrift von einer einfachen Organisation, die zwischen Verlag und Grundversorgergesellschaften höchste Effizienz und Mitsprachemöglichkeiten ergibt. Einzigartig dürfte auch die offene Abrechnung der Zeitschrift sein, die den beteiligten Fachgesellschaften ein Partizipieren am Gewinn der Zeitschrift erlaubt (siehe PrimaryCare 2001;1:230-7).

PrimaryCare wird im Verlag von Dr. Natalie Marty (Chefredaktorin), Dr. Romaine Viollier (stellvertretende Chefredaktorin) und Louise Bigler (Sekretariat) betreut. Die sehr aktive Redaktion besteht aus 6 Vertreterinnen und Vertretern der SGAM und 2 Vertre-

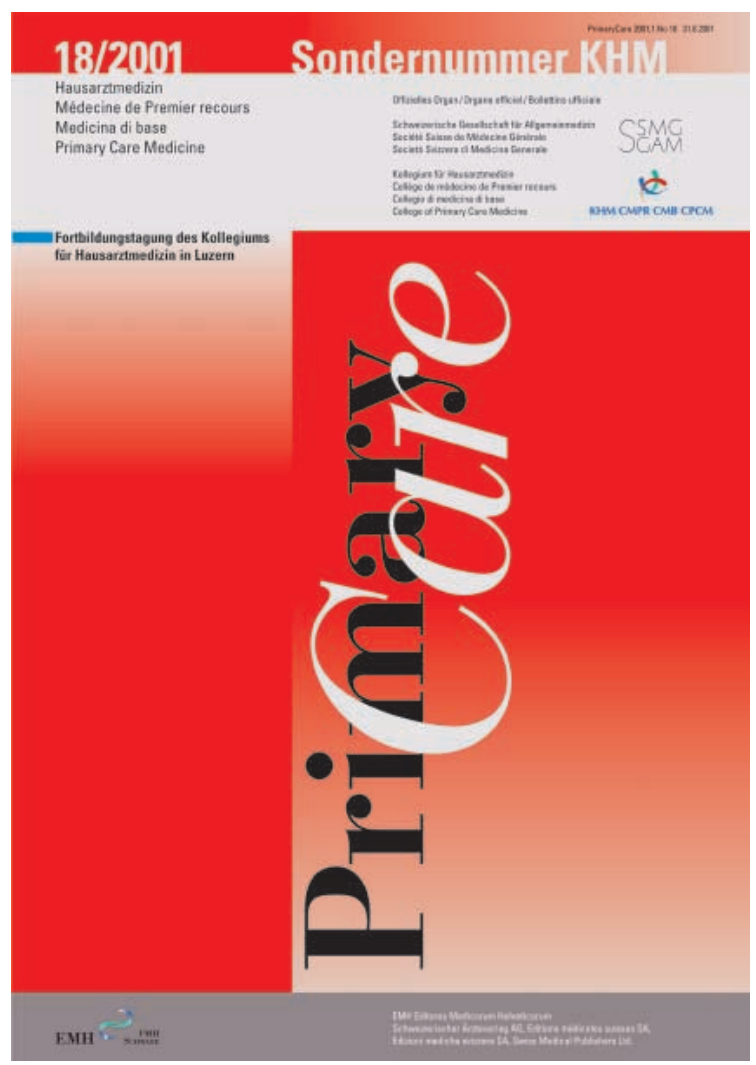

terinnen und Vertretern des KHM: Dr. Heinz Bhend, Dr. Silva Keberle, Dr. Bruno Kissling, Dr. Pierre Klauser, Dr. François Mottu, Dr. Bernhard Rindlisbacher und Dr. Daniel Widmer.

PrimaryCare erscheint 14täglich und publiziert standespolitische Beiträge und Artikel im Bereich der Fortbildung und Qualitätsförderung. Die Zeitschrift entwickelt sich sehr rasch zu einer lebendigen Diskussionsplattform für die Anliegen der Grundversorgerinnen und Grundversorger in der Schweiz. Glaubwürdigkeit bezieht PrimaryCare aus dem redaktionellen Grundsatz, das verbreitete Prinzip des "Artikels zum Inserat" absolut auszuschliessen. Auch bei medizinischen Fortbildungsbeiträgen wird Unabhängigkeit und Neutralität absolute Priorität eingeräumt.

\section{Schweizerisches Medizinisches Jahrbuch}

Das Schweizerische Medizinische Jahrbuch ist erstmals nicht nur als Printprodukt, sondern auch im Internet vorhanden. Neben der Publikation sämtlicher Adressen der Mitglieder von FMH, SSO und des Schweizerischen Apothekervereins ist vor allem der "Spitalteil» vollkommen überarbeitet und mit neuen Informationen, detailliert bis auf Abteilungsstufe, im Internet vorhanden. Erfreulich ist die aktive Mitarbeit der einzelnen Spitäler und Gesundheitsorganisationen. Das Online-Projekt ist mit Erfolg aufgeschaltet worden. Allerdings stehen wir bei der Publikation dieses elektronischen Produkts erst am Anfang der Entwicklung. Hier können bisher völlig unbekannte Informationen zugänglich gemacht und interessante 


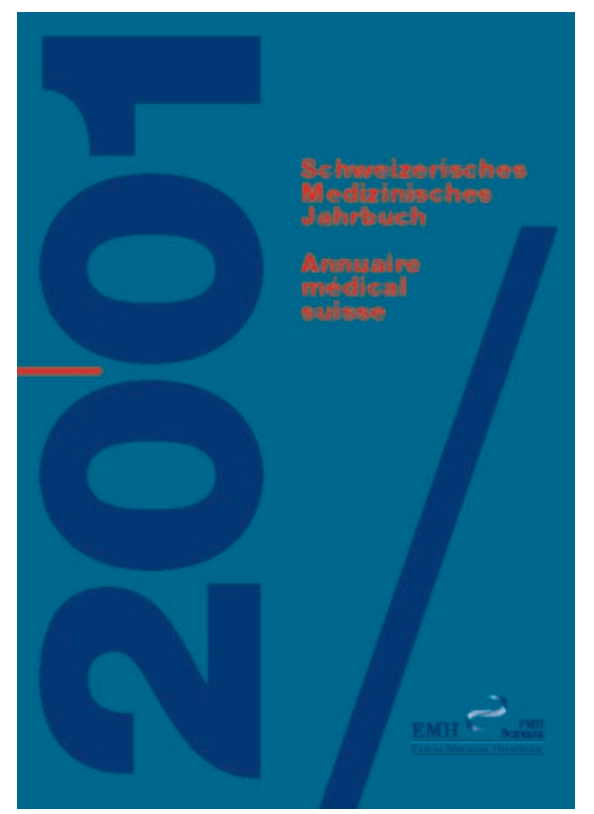

Abfragen gemacht werden. Allerdings ist eine grosse Subtilität im Umgang mit diesen Daten gefordert, und die Massgaben des Datenschutzes sind konsequent zu berücksichtigen.

\section{Kardiovaskuläre Medizin}

Die «Kardiovaskuläre Medizin» erscheint unter der Schriftleitung von Prof. Thomas F. Lüscher, Zürich, und Prof. René Lerch, Genf, seit 1997. Sie widmet sich

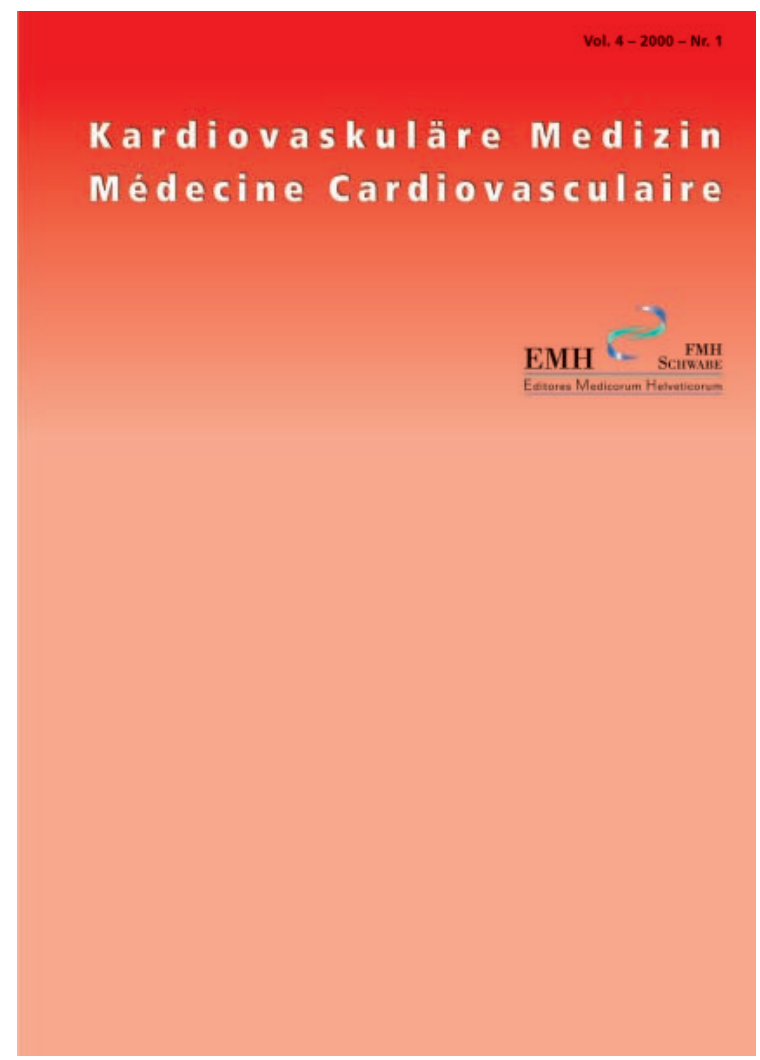

der gesamten Breite der kardiovaskulären Medizin und ist daher ein optimales Forum für Fortbildung, wissenschaftliche Arbeiten im Bereiche Kardiologie, Angiologie, Hypertonie sowie Herz- und Gefässchirurgie. Neben pointierten Editorials und klinischen Originalarbeiten und Kongressberichten bietet die Zeitschrift Mitteilungen und - gewissenmassen aus der Praxis für die Praxis - "Images in Cardiovascular Medicine" mit ungewöhnlichen Befunden verschiedener bildgebender Verfahren, "Das interessante EKG» sowie einen Terminkalender für kardiologische Fortbildungsveranstaltungen und Kongresse.

\section{Synapse}

Die Synapse ist das offizielle Kommunikationsorgan der Ärztegesellschaft Baselland. Dieses Jahr hat Dr. Franz Rohrer die Leitung der Redaktion von Dr. Lukas Wagner übernommen. Die Synapse trägt das grosse Potential einer basisnahen und regionalen Informationspolitik, welche die Ärztegesellschaft Baselland konsequent weiterführen wird; sie darf dabei auf eine in allen Belangen professionelle Unterstützung durch den Schweizerischen Ärzteverlag zählen.

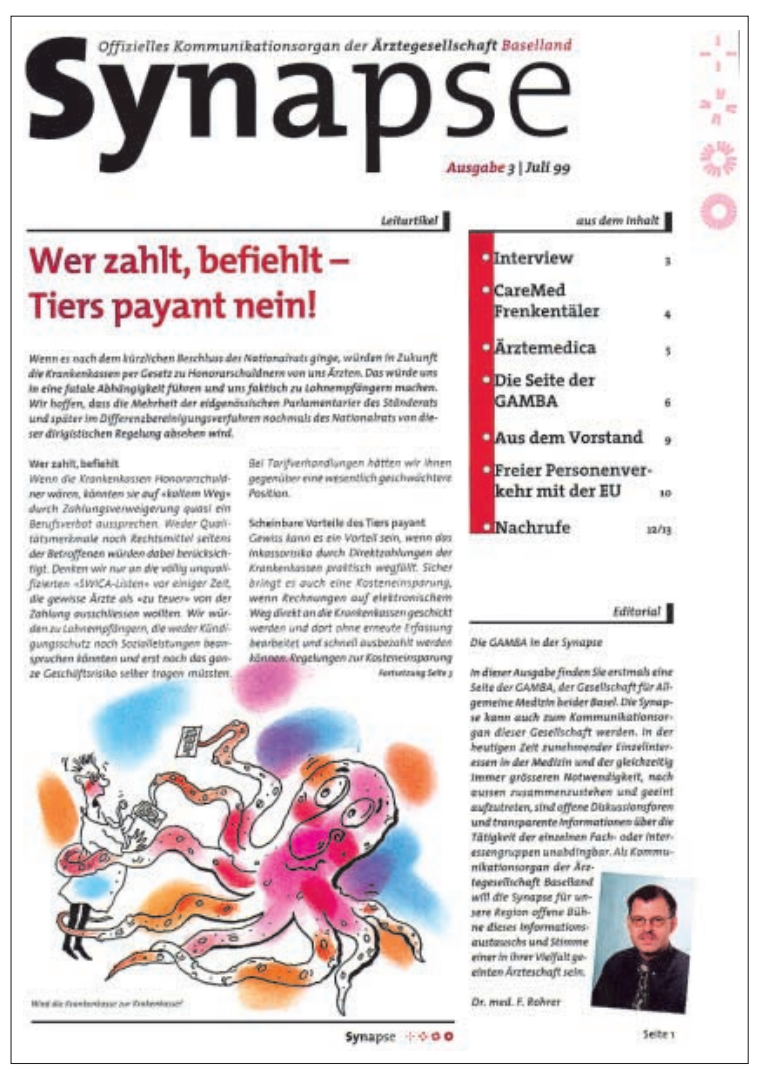

\section{medkalender}

Der medkalender konnte wegen der lange Zeit unklaren Preisgestaltung im Medikamentenmarkt nicht wie vorgesehen publiziert werden. Eine neue Auflage wird geplant. 


\section{Personalsituation}

Mit einiger Verzögerung sind nun alle geplanten Stellen besetzt. Die verspätete Besetzung einiger Stellen hat einerseits zu beträchtlichen Kosteneinsparungen im Berichtsjahr, andererseits zu grosser Belastung der Mitarbeiter/innen geführt, die an der Aufbauarbeit beteiligt waren. Das EMH-Team wird neuerdings verstärkt durch eine zusätzliche Lektorin (Dr. med. Romaine Viollier) sowie eine Marketing-/Internetspezialistin (Natacha Gerber). Ausserdem bildet der Schweizerische Ärzteverlag mit Roland Bienz einen KV-Lehrling mit Schwerpunkt Informatik aus.

\section{Aktuelle Buchprojekte}

Eine Buchreihe zu Themen der Gesundheitsökonomie ist geplant. Sie wird im Verlag durch Dr. Markus Trutmann betreut. Als Beirat konnten alle prominenten Gesundheitsökonomen der Schweiz gewonnen werden. Der erste Band ist in Produktion. legt.

Das Manual der Vertrauensärzte wird neu aufge-

\section{EMH im Internet}

Die Aktivitäten im Bereich Internet wurden einerseits vom Aufbau der EMH-Website (www.emh.ch), die nach einer insgesamt sechsmonatigen Planungsund Realisierungsphase termingerecht per Ende 2000 aufgeschaltet werden konnte, andererseits von den Auftritten der neuen Verlagspublikationen geprägt.

Nach der Online-Version der Schweizerischen Ärztezeitung (www.saez.ch) im Juni 2000 war die EMH-Website das grösste Internetprojekt des Verlages im Berichtsjahr. Es vereint einerseits die verschiedenen Online-Versionen der Verlagspublikationen, andererseits wird der zunehmende Bedarf an allgemeiner Information abgedeckt. Längerfristig soll die Website als Portal für die Ärzteschaft der Schweiz die "Marke EMH» mitdefinieren.

www.emh.ch bietet in der Rubrik "News" im Zusammenarbeit mit infomed laufend aktuelle Links zu wichtigen Volltextpublikationen im Internet. Der Verlag infomed ist seit Jahren eine sichere Adresse für zuverlässige Informationen, und wir freuen uns besonders, dass die gute Zusammenarbeit in diesen neuen Bereich ausgedehnt werden konnte. Für den Cartoon des Tages können wir mit Randy Glasbergen, einem der weltbekannten Cartoonisten, täglich ein "Zückerli» hinzufügen. Die News-Rubrik soll stark ausgebaut werden, ohne aber $\mathrm{zu}$ einem blinkenden Mischmasch von Pressecommuniqués zu verkommen.

In der Rubrik "Medical addresses" findet sich der gesamte Bestand der FMH-Mitgliederadressen. Mit einem Feedbackformular trägt dieser "Ärzteindex" dazu bei, die Daten der FMH aktuell zu halten. Weiter sind unter "Hospitals» die Spitäler der Schweiz bis auf Abteilungsebene abrufbar - diese Spitaldaten-
Jürg H.Sommer

Muddling Through Elegantly: Rationierung im Gesundheitswesen

bank ist einmalig und wurde von Grund auf von EMH entwickelt.

In der Rubrik "Congresses" sind die Kongressdaten aus EMH-Kongressdatenbank komplett abrufbar, die den Leserinnen und Lesern auch als Beilage der Schweizerischen Ärztezeitung bekannt ist. Veranstaltungen können durch verschiedene Suchfunktionen aufgefunden werden. Ein Anmeldeformular ermöglicht einen kostenlosen Direkteintrag im Kongresskalender.

Unter "EMH interactive» befindet sich ein allgemeines Diskussionsforum zu gesundheitspolitischen Themen. In den Zeitschriftenforen sind jeweils Kommentare und Leserbriefe zu Artikeln aus unseren Zeitschriften publiziert.

Ein wichtiger Bestandteil unseres Internetkonzeptes besteht darin, den einzelnen Publikationen eigene prägnante Internetauftritte mitzugeben.

Unter www.emh.ch sind allgemeine Informationen zu den Zeitschriften einsehbar. Mit einem Link wird auf den entsprechenden Auftritt verwiesen.

- die Schweizerische Ärztezeitung ist seit dem 21. Juni 2000 online (www.saez.ch). Die Artikel erscheinen synchron zur gedruckten Ausgabe im Volltext. Umfassende Suchfunktionen geben der Website Archivcharakter.

- Der SMW (www.smw.ch) wurde aufgrund der Namens- und Konzeptänderung ein neues Kleid verpasst. Die Schweizerische Medizinische Wochenschrift war als eine der ersten deutschspra- 
chigen Medizinzeitschriften schon seit 1996 im Netz. Alle Artikel ab 1999 sind im Volltext verfügbar. Entsprechend hoch sind die Zugriffszahlen bei diesem Angebot (etwa 1000 Benutzer pro Tag). Für die "neue" SMW (Swiss Medical Weekly) als englischsprachiges Fachorgan ist für das Medium Internet zentral. Wir verfolgen die Entwicklung der grossen medizinischen Datenbanken aufmerksam und werden in Zukunft die SMW neben der Medline auch in die Volltextdatenbanken integrieren.

- Die Website des "Swiss Medical Forum" (www. medicalforum.ch) wurde gleichzeitig zur gedruckten Version lanciert und bietet wie alle EMH-Titel interessante Suchfunktionen. Noch wichtiger ist aber hier das grosse Selbstevaluationsprojekt, das wir mit einer eigenen Softwarelösung demnächst lancieren können.

- Auch alle Artikel der Zeitschrift "PrimaryCare» finden sich seit dem Start auf der entsprechenden Website (www.primary-care.ch). Die Redaktion arbeitet eng mit der Redaktion der SGAM-Website www.sgam.ch/www.ssmg.ch zusammen.

All diese Angebote können direkt von www.emh.ch angewählt werden. Redaktionell betreute Linklisten, die direkte Suche in der Medline und ein Online-Shop runden dieses Angebot ab. 